\title{
Single-Stranded Conformation Polymorphism (SSCP) Analysis
}

National Cancer Institute ( $\mathrm{NCl})$

\section{Source}

National Cancer Institute (NCI). Single-stranded conformation polymorphism analysis.

A laboratory test used to separate single-stranded nucleic acids based on subtle differences in their DNA sequence, often a single base pair, which results in a different secondary structure and a measurable difference in mobiity through a gel. Also called SSCP analysis. 